\title{
CAPTURING ARTICULATION IN ASSEMBLIES FROM COMPONENT GEOMETRY
}

\author{
Rajarishi Sinha ${ }^{1}$ \\ Institute for Complex Engineered Systems \\ 1212 Hamburg Hall, Carnegie Mellon University \\ Pittsburgh, PA 15213 \\ Email: rsinha+@cmu.edu \\ Tel.: 412-268-5214 \\ Satyandra K. Gupta \\ Robotics Institute \\ Carnegie Mellon University \\ Pittsburgh, Pennsylvania 15213 \\ Email: skgupta@ri.cmu.edu
}

\author{
Christiaan J.J. Paredis \\ Institute for Complex Engineered Systems \\ Carnegie Mellon University \\ Pittsburgh, Pennsylvania 15213 \\ Email: paredis+@cmu.edu \\ Pradeep K. Khosla \\ Institute for Complex Engineered Systems \\ Carnegie Mellon University \\ Pittsburgh, Pennsylvania 15213 \\ Email: pkk@cs.cmu.edu
}

\begin{abstract}
This paper presents a method to extract instantaneous articulation from part geometry, based on surface mating constraints as well as constraints imposed by other incidental contacts.

Many assemblies contain joints, each of which have degrees of freedom associated with them. These joints allow the relative positions of parts in the mechanism to change as the joints are articulated. Being able to represent these joints and their behavior is important from the designers perspective because it enables him or her to verify whether kinematic requirements have been met.Therefore, it is useful to be able to obtain such joint information directly from part geometry and contact physics.

The method presented here handles all lower pairs of kinematic joints. Surface mating contacts are classified into one of three types: planar, spherical and cylindrical. The contacts are represented by algebraic inequalities describing the translational and angular velocities at the contact. Non-penetration conditions are written for a finite set of points on the boundary of each contact face, and it is shown that the finite set of conditions is representative of the entire boundary and the region enclosed by the boundary. Simultaneous satisfaction of the non-penetration conditions at all the contact surfaces between a pair of bodies is represented by a 6 -dimensional simplex, which can be solved using linear programming.
\end{abstract}

${ }^{1}$ Author to whom all correspondence should be addressed.

\section{INTRODUCTION}

Assemblies are composed from parts. The geometry of the parts imposes certain restrictions on the way that they can be assembled, and also on the way that they move relative to one another. The joints between parts are defined by the designer at the conceptual design level to meet certain functional requirements of the assembly. Thus, there can be two types of constraints between parts, namely, constraints induced by the geometry of parts, and constraints introduced by the designer to satisfy functional requirements of the assembly. Both these types of constraints interact to produce a resultant behavior of a joint.

At the conceptual level, the designer knows the type and behavior of the joints in the concept design. However, the geometry still needs to be defined. At the preliminary design stage, the geometric information is defined, and the relative positions and orientations of components in the assembly are specified. Following this stage, component and joint representations are enriched to achieve a final refinement of the geometry.

In order to generate assembly or disassembly plans for such assemblies, the designer needs to take articulation information into consideration. However, current methods 
of representing articulation are restricted to systems which require complete specification by the user or are feature recognition based. The former are open to incorrect input by the user resulting in illegal articulation behavior. The latter do not account for incidental contacts.

In this paper, we present a methodology that extends earlier work on planar contact surfaces and reasons about the degrees of freedom at each joint based on surface mating constraints, which are in turn obtained from analyzing the nature of body to body contact. Non-penetration constraints are imposed along the boundary of each contact surface in the form of algebraic inequalities. It is shown that a finite number of non-penetration conditions are representative of the entire surface in contact. Using linear programming methods, instantaneous velocities and accelerations for each pair of bodies are computed. This determines the type of joint and its features.

The algorithms and analysis presented here apply to the so-called "lower" kinematic pairs, namely, the Revolute, Cylindrical, Planar, Prismatic and Spherical joint types. The types of surfaces in contact must be planar, cylindrical or spherical. Since linear programming techniques are used to find a solution, the number of constraints must be finite. We will show that for this reason, some types of patch boundaries must be approximated as "straight line segments" in its topology (straight lines on the plane; circular arcs or vertical lines on the cylinder; great arcs on the sphere).

Such a methodology is useful in that it can provide useful feedback to the designer. He or she can determine which components are free to move in the assembly. The procedure can be completely automated, so that there is no user interaction. This eliminates the possibility of input errors. In addition, since the method is algebraic and uses linear programming, it is extremely fast and is valid for all possible surface contacts which fall into one of three classes, unlike other rule-based systems which operate on a feature level. This method will also account for contact surfaces with incomplete geometry (such as portions of planes, cylinders or spheres).

\section{REVIEW OF PREVIOUS WORK}

\section{Overview}

Assembly modeling has been studied extensively. In their survey, Sodhi and Turner (1994) argue that a CAD environment for design should have the ability to represent the design in multiple ways and the ability to link these representations to the functionality of the product. Functionality should be preserved after changes in the design.
Work in the area of representing and deriving articulation in assemblies can be broadly classified into three groups: rule-based methods, algebraic methods, and grouptheoretic methods.

The earliest works on assembly modelling (cf. (Ambler and Popplestone, 1975)) presented the definitions for part mating constraints. Tilove (1983) described a method to define links and joints to support animation of assemblies. Thomas and Torras (1992) reported a method to determine the final relative positions of parts in an assembly given the geometry of the parts and the mating constraints. Turner et al. (1992) present a method to obtain the degrees of freedom of a part in an assembly from its mating constraints. Mattikalli and Khosla (1991) describe a method to obtain degrees of freedom from component mating constraints, wherein they use a unit sphere to represent the space of all available degrees of freedom. Wilson and Latombe (1994) introduce the concept of a non-directional blocking graph which describes the allowed degrees of freedom after surface mating constraints have been considered. Anantha et al. (1996) describe assembly modelling by the satisfaction of geometric constraints.

Ge and McCarthy (1991) characterize the space of possible relative positions of two components using the Clifford Algebra. Liu and Popplestone (1994) present a method to describe surface contacts in terms of symmetry groups.

Other researchers have presented work on assembly modelling systems ( (Tilove, 1983), (Ko and Lee, 1987), (Kim and Lee, 1989), (Rajan and Nof, 1996)) which handle mating conditions and kinematic simulation. Rocheleau (1996) described an assembly representation which used connectivity sets to store assembly data hierarchically and including pairwise topological relationships.

Some issues have not yet been addressed satisfactorily. Current shortcomings in articulation research include:

1. User-defined articulation is time consuming and prone to errors.

2. Many representations are tool-specific (ADAMS, Pro/ENGINEER etc). One has to model the part geometry and the mating surface constraints in the same CAD tool; model export or model translation does not retain articulation information.

3. Current representation is purely geometry-based without significant physics-based reasoning.

4. Current techniques are local; global interaction (propagation of constraints beyond the point where they are induced) is not satisfactory.

5. Current simulation techniques do not detect incorrect/incomplete inputs; editing parts may make the simulation return incorrect results. There is no verification for correctness of the articulation representation 
and for the compound effect of geometric interactions and physics-based interactions.

This paper falls into the second category - that of algebraic methods and will attempt to advance the state of the art with regard to all five shortcomings listed above.

\section{Contact Mechanics for Planar Contacts}

A part in an assembly is in physical contact with one or more other parts. The nature of these contacts can provide useful information about the types and limits of the degrees of freedom at these contact points. Some of these contacts induce surface mating constraints, leading to the formation of a joint. Other contacts are incidental, in that they may introduce limits on the degrees of freedom of the joint (Rajan et al., 1997). Reasoning about these constraints provides the designer with valuable insight into the instantaneous degrees of freedom of the assembly.

Other researchers ((Mattikalli et al., 1994), (Baraff and Mattikalli, 1993)) have worked with polygonal bodies and polygonal surfaces of contact. They approximate curved planar boundaries using straight lines, and use linear programming techniques to solve the contact problem.

When a pair of parts are in contact with each other, it implies that there is no inter-penetration between the parts at the contact surfaces. This non-penetration condition at a point can be written as (Baraff and Mattikalli, 1993):

$$
(\vec{v}+\vec{\omega} \times \vec{r}) \cdot \vec{n} \geq 0
$$

where $\vec{v}$ is the translational velocity, $\vec{\omega}$ is the angular velocity, $\vec{r}$ is the position of the point and $\vec{n}$ is the normal at a point of contact on the surface of contact. This equation is linear in $\vec{v}$ and $\vec{\omega}$. It implies that the generalized velocity vector for relative motion between two points, one on each contact surface, should not have a component opposite to the normal to the base surface. A component into the base surface will imply penetration.

To prevent the penetration of one part into the other, Equation (1) must be satisfied at every point on the surface of contact and on the boundary of the surface of contact.

Consider a straight line segment on a plane. The nonpenetration conditions at the end-points of the segment can be written as:

$$
\begin{aligned}
& \left(\vec{v}+\vec{\omega} \times \overrightarrow{r_{1}}\right) \cdot \vec{n} \geq 0 \\
& \left(\vec{v}+\vec{\omega} \times \overrightarrow{r_{2}}\right) \cdot \vec{n} \geq 0
\end{aligned}
$$

If inter-penetration does not occur at the end points, then it will not occur anywhere on the line segment $1 \rightarrow$

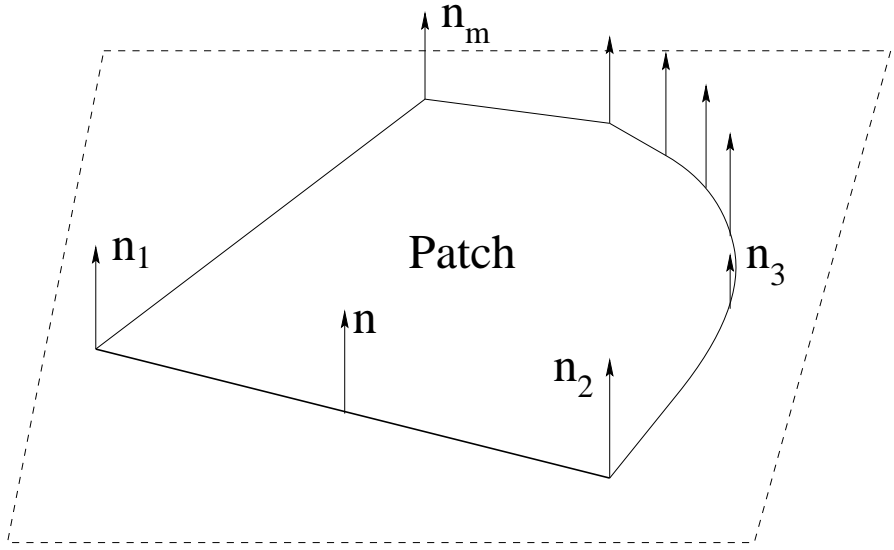

Figure 1. Plane Patch with Straight and Curved Line Segments on the Boundary.

2 (Figure 1). We can prove this by multiplying the first inequality by $x_{1}$ and the second by $x_{2}$ and rearranging terms to get:

$$
x_{1}\left(\left(\vec{v}+\vec{\omega} \times \overrightarrow{r_{1}}\right) \cdot \vec{n}\right)+x_{2}\left(\left(\vec{v}+\vec{\omega} \times \overrightarrow{r_{2}}\right) \cdot \vec{n}\right) \geq 0
$$

or:

$$
\left(\left(x_{1}+x_{2}\right) \vec{v}+\vec{\omega} \times\left(x_{1} \overrightarrow{r_{1}}+x_{2} \overrightarrow{r_{2}}\right)\right) \cdot \vec{n} \geq 0
$$

Equation 4 can be written in this way because the normal $\vec{n}$ to a planar body at any point on the body is a constant. Equation 4 has the same form as Equation 1 when $x_{1}+x_{2}=1$ with $x_{1}$ and $x_{2} \geq 0$ and $r=$ $x_{1} r_{1}+x_{2} r_{2}$. Therefore, the necessary and sufficient condition for non-penetration along the straight line segment is non-penetration at the end points.

Extending this result to planar polygonal bodies is done in a similar fashion, resulting in the fact that interpenetration at any point in the interior or on the boundary of a polygon will not occur if inter-penetration does not occur at the vertices. Such a method of expressing planar contact between two bodies has been used before. For example, see Baraff and Mattikalli (1993), where the authors use non-penetration conditions to determine the impending motion direction of rigid bodies in contact.

This analysis deals with surface contacts which fall into one of three categories, namely, planar, cylindrical, or spherical. One or more such contacts give rise to the lower kinematic joint pairs. However, it is not practical to write Equation 1 at every point in the interior of the surface of contact. We will show that is sufficient to write the non-penetration 
condition for a finite number of points on the boundary of the contact surface, under certain conditions.

A closed planar surface (or patch) is bounded by a finite set of curves; these curves may be straight line segments or curved line segments. Any point in the interior of the patch can be expressed as a linear combination of points at the vertices of the boundary of the convex hull of the patch. As long as the number of vertices is finite, there will be a finite number of non-penetration conditions, all of which will be linear in $\vec{v}$ and $\vec{\omega}$.

To ensure that there are a finite number of vertices, we discretize the curved line segments on the boundary by straight line segment approximations (Figure 1).

\section{CONTACT MECHANICS FOR SPHERICAL AND CYLINDRI- CAL SURFACES}

In this section, we extend the results obtained for planar surface contacts by showing that similar results can also be obtained for spherical and cylindrical surfaces defined by edges which are great arcs (for spherical surfaces) or straight lines and circular arcs (for cylindrical surfaces).

\section{Spherical Contacts}

Spherical surfaces in contact always have unconstrained rotations, because they share a common center. As before, the non-penetration conditions must be written at the vertices of the convex cone for the given spherical contact surface. However, since the cost of computing the convex cone is high, we choose to generate the non-penetration condition at the vertices of the spherical patch. This will not influence the final result. The non-penetration conditions at two points on a sphere are written (in a fashion similar to Equation 1) as:

$$
\begin{aligned}
& \left(\vec{v}+\vec{\omega} \times\left(\vec{p}+R \overrightarrow{n_{1}}\right)\right) \cdot \overrightarrow{n_{1}} \geq 0 \\
& \left(\vec{v}+\vec{\omega} \times\left(\vec{p}+R \overrightarrow{n_{2}}\right)\right) \cdot \overrightarrow{n_{2}} \geq 0
\end{aligned}
$$

Using techniques similar to those used for planar patches, i.e. multiplying the first inequality by $x_{1}$ and the second by $x_{2}$, and then adding, we get:

$$
\left(x_{1} \overrightarrow{n_{1}}+x_{2} \overrightarrow{n_{2}}\right) \cdot(\vec{v}+\vec{\omega} \times \vec{p}) \geq 0
$$

where the left hand side term is an expression of $\vec{n}$ as a linear combination of $\overrightarrow{n_{1}}$ and $\overrightarrow{n_{2}}$ for $x_{i}>0 \forall i=1 \ldots 2$. The $\vec{n} \cdot \vec{\omega} \times \vec{n}$ term is always 0 for all $\vec{n}$, and drops out of the expression. Therefore, inter-penetration does not occur at any point within the patch (Figure 2).

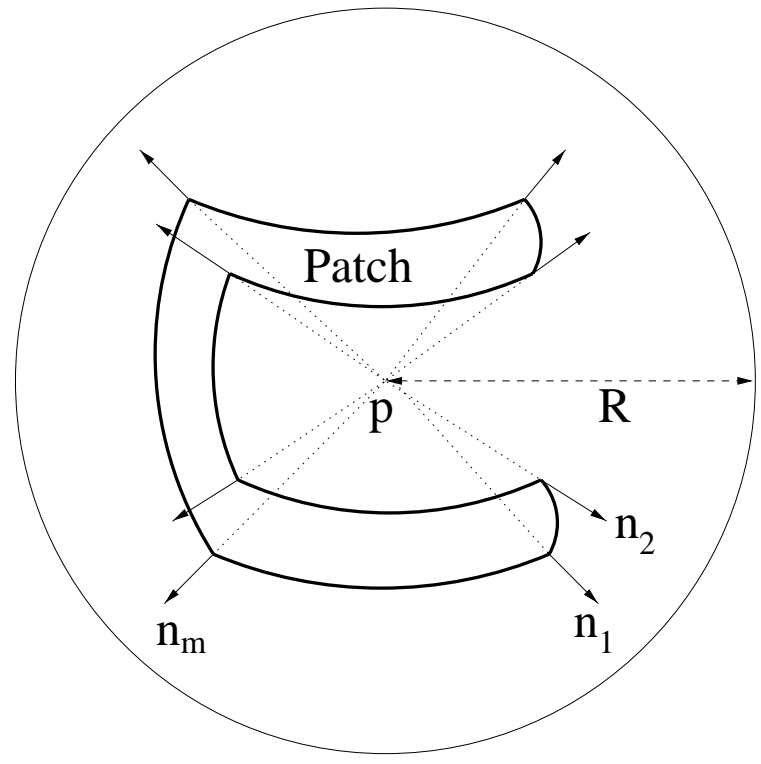

Figure 2. Spherical Patch with Great Arc Boundary Segments.

Boundary segments of the spherical patch must be discretized into a finite number of great arcs so that nonpenetration conditions can be imposed at each end-point of the arcs. When the angle subtended by a great arc at the center is greater than $\pi$, then a third point should be picked in between the end points such that the angle between the first and third points as well as the angle between the second and third points are both less than $\pi$. This will ensure that the normals at the first and third points span the circular arc between the first and third points. Similarly, the normals at the second and third points span the circular arc between the second and third points. The non-penetration condition for the third point should be added to the set of non-penetration conditions for this patch.

\section{Cylindrical Contacts}

Cylindrical surface contacts, by virtue of the fact that the contact surface is curved, do not have a normal vector that is constant over the entire patch. Thus, we define a normal with the following property:

$$
\vec{n} \cdot \vec{o}=\overrightarrow{0}
$$

with the origin of the cylinder at $\vec{p}$ and $\vec{o}$ along the axis of the cylinder of which the patch is a part (Figure 3). Now, the non-penetration conditions at two points 1 and 2 on the patch becomes:

$$
\overrightarrow{n_{1}} \cdot\left(\vec{v}+\vec{\omega} \times\left(\vec{p}+z_{1} \vec{o}+R \overrightarrow{n_{1}}\right)\right) \geq 0
$$




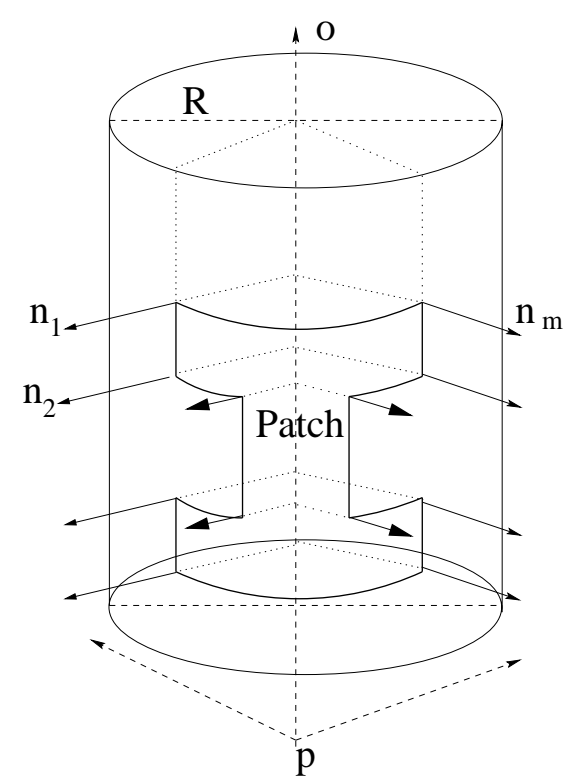

Figure 3. Cylindrical Patch with Boundary Segments.

$$
\overrightarrow{n_{2}} \cdot\left(\vec{v}+\vec{\omega} \times\left(\vec{p}+z_{2} \vec{o}+R \overrightarrow{n_{2}}\right)\right) \geq 0
$$

where $\vec{p}$ is the origin of the cylinder, $z_{i}$ is the distance from the origin to the point in question, along $\vec{o}$ and $R$ is the radius of the cylinder of which the patch is a part.

Equation 8 should hold at every point on the cylindrical patch for the non-penetration condition to be valid.

Multiplying each inequality by $x_{1}, x_{2}$ and so on, and then adding results in:

$$
\begin{aligned}
& \left(x_{1} \overrightarrow{n_{1}}+x_{2} \overrightarrow{n_{2}}\right) \cdot(\vec{v}+\vec{\omega} \times \vec{p})+ \\
& \quad\left(x_{1} z_{1} \overrightarrow{n_{1}}+x_{2} z_{2} \overrightarrow{n_{2}}\right) \cdot(\vec{\omega} \times \vec{o}) \geq 0
\end{aligned}
$$

During the consideration of each patch boundary segment, it is necessary to show that there exist $\vec{n}=x_{1} \overrightarrow{n_{1}}+x_{2} \overrightarrow{n_{2}}$ and $z \vec{n}=x_{1} z_{1} \overrightarrow{n_{1}}+x_{2} z_{2} \overrightarrow{n_{2}}$ with $\vec{n} \cdot \vec{o}=0$ for the segment.

Considering Boundary Points Constant in $z$. To show that for a boundary segment with constant $z$, the $\theta$ coordinate of a point on the boundary can be expressed as a linear combination of the $\theta$ coordinates of the end points, we set $z_{1}=z_{2}=\ldots=z$ in Equation 9 to get:

$$
\vec{n}=\frac{x_{1} \overrightarrow{n_{1}}+x_{2} \overrightarrow{n_{2}}}{\left\|x_{1} \overrightarrow{n_{1}}+x_{2} \overrightarrow{n_{2}}\right\|}
$$

Points which subtend an angle at the axis which is greater than $\pi$ are resolved by considering an additional point at the middle of the circular contact arc between these two points. Then the angle between each of the original points and the newly added point will be less than $\pi$. From examination of Figure 3, we can see that the normal vectors at two points with the same $z$ and different $\theta$ (such as the segment from $\overrightarrow{n_{m}}$ to $\overrightarrow{n_{1}}$ ) span the possible normals between the two points. Therefore the non-penetration condition at any point on a boundary segment with constant $z$ is satisfied if non-penetration is satisfied at the end points of the segment.

Considering Boundary Points Constant in $\theta$. To show that for a boundary segment with constant $\theta$, the $z$ coordinate of a point on the boundary can be expressed as a linear combination of the $z$ coordinates of the end points, we set $\overrightarrow{n_{1}}=\overrightarrow{n_{2}}=\vec{n}$ in Equation 9 to get:

$$
\begin{aligned}
z & =x_{1} z_{1}+x_{2} z_{2} \\
x_{1}+x_{2} & =1
\end{aligned}
$$

From examination of Figure 3, we can see that the normal vectors at two points with the same $\theta$ and different $z$ (such as the segment from $\overrightarrow{n_{1}}$ to $\overrightarrow{n_{2}}$ ) have normals which are equal. Thus, these two points are very similar to the end-points of a straight line in a plane. Therefore the nonpenetration condition at any point on a boundary segment with constant $\theta$ is satisfied if non-penetration is satisfied at the end points of the segment.

Considering Boundary Points Varying in $z$ and $\theta$. For situations when the points on the boundary of the patch vary both in $z$ and in $\theta$, it is impossible to find an $\vec{n}=x_{1} \overrightarrow{n_{1}}+x_{2} \overrightarrow{n_{2}}$ such that $x_{1} z_{1} \overrightarrow{n_{1}}+x_{2} z_{2} \overrightarrow{n_{2}}=z \vec{n}$. So, we discretize the boundary using segments of constant $z$ and constant $\theta$. This ensures that along each discretized segment, the linear relationship between the end points holds.

\section{SOLVING FOR INSTANTANEOUS ARTICULATION}

\section{Solving the Set of Non-Penetration Conditions}

Each primitive patch induces a non-penetration condition at each of its (finite) vertices. Since inter-penetration must not occur at any point at any time, the inequalities for all the non-penetration conditions for the all the patches of a pair of bodies considered simultaneously form the linear program:

$$
\overrightarrow{n_{i}} \cdot\left(\vec{v}+\vec{\omega} \times \overrightarrow{r_{i}}\right) \geq 0
$$


where $i=1 \ldots$ Total number of vertices in all patches, $\overrightarrow{n_{i}}$ is the normal at each vertex and $\overrightarrow{r_{i}}$ is the position of each vertex.

The simplex for this program is 6 -dimensional (3 variables for translational velocity and 3 variables for angular velocity).

\section{Global Solution for Instantaneous Articulation}

Since at any time, all the non-penetration conditions for all the parts must be satisfied, it is possible to solve all the inequalities for all the vertices of all the parts in the same linear program. This will result in a solution which is globally valid. Using a single linear program, it is possible to obtain all the instantaneous degrees of freedom for the assembly. If we write the non-penetration condition for a patch concisely as:

$$
\mathbf{J}_{\text {patch }}\left[\begin{array}{c}
\vec{v} \\
\vec{\omega}
\end{array}\right] \geq 0
$$

where $\mathbf{J}_{\text {patch }}$ is the Jacobian for that particular patch. Then the non-penetration conditions for all the patches in a bodybody contact pair formed between bodies $A$ and $B$ can be written as:

$$
\mathbf{J}_{A B}\left[\begin{array}{c}
\overrightarrow{v_{A}}-\overrightarrow{v_{B}} \\
\overrightarrow{\omega_{A}}-\overrightarrow{\omega_{B}}
\end{array}\right]=\left[\begin{array}{c}
\mathbf{J}_{\text {patch }_{1}} \\
\mathbf{J}_{\text {patch }_{2}} \\
\vdots \\
\mathbf{J}_{\text {patch }_{p}}
\end{array}\right]\left[\begin{array}{c}
\overrightarrow{v_{A}}-\overrightarrow{v_{B}} \\
\overrightarrow{\omega_{A}}-\overrightarrow{\omega_{B}}
\end{array}\right] \geq 0
$$

where $p$ is the number of patches in which this body-body pair participates. Using expressions such as Equation 14 written for all the body-body contact pairs in an assembly, we get:

$$
\begin{gathered}
\mathbf{J}_{\text {assembly }}\left[\begin{array}{c}
\overrightarrow{v_{A}}-\overrightarrow{v_{B}} \\
\overrightarrow{\omega_{A}}-\overrightarrow{v_{B}} \\
\overrightarrow{v_{B}}-\overrightarrow{v_{C}} \\
\vdots
\end{array}\right]= \\
{\left[\begin{array}{ccccc}
\mathbf{J}_{A B} & -\mathbf{J}_{A B} & 0 & \ldots & 0 \\
0 & \mathbf{J}_{B C} & -\mathbf{J}_{B C} & \ldots & 0 \\
\vdots & \vdots & \vdots & \ddots & \vdots
\end{array}\right]\left[\begin{array}{c}
\overrightarrow{v_{A}}-\overrightarrow{v_{B}} \\
\overrightarrow{\omega_{A}}-\overrightarrow{\omega_{B}} \\
\overrightarrow{\omega_{B}}-\overrightarrow{\omega_{C}} \\
\vdots
\end{array}\right] \geq 0}
\end{gathered}
$$

$\mathbf{J}_{\text {assembly }}$ is a complete representation of the assembly with instantaneous articulation. Solving this global simplex

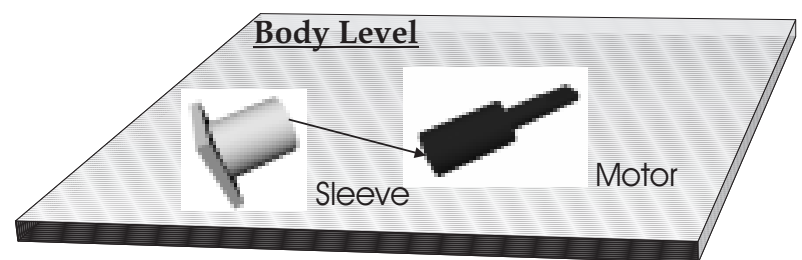

Figure 4. The Articulation Representation Schema.

provides all the translational and angular velocities for all the body-body pairs simultaneously.

\section{RELATING THE THEORY TO A CAD ENVIRONMENT}

\section{Interfacing with CAD Models}

Beginning from a very high level representation of the assembly, we proceed to progressively more detailed representations of the instantaneous articulation (Figure 4).

A contact graph structure can be used to represent the assembly. In the contact graph, parts are represented as nodes, and contacts between parts are represented as edges between the corresponding nodes. Edges between nodes are automatically derived by performing intersections between the nodes. Each part is scaled by a measure proportional to its bounding box dimensions, so that the result of the intersection is a regular solid (or a set of regular solids). 
In each pair of nodes, there is one which is a base part, and another which is an attached part. The contact graph can be represented as:

$$
\begin{aligned}
G= & \{(A, B, I): A, B \in \text { Set of parts; } A \neq B \\
& I=\text { Result of intersection between } \mathrm{A} \text { and } \mathrm{B}\}
\end{aligned}
$$

The edges still remaining in the tree form candidates for joints. At this stage, the intersection information in each edge is examined for features which could indicate the presence of surface mating constraints.

Each element of $I$ can be thought of as a constraint patch on the mating surface between $A$ and $B$. $I$ can be partitioned into three disjoint sets, for cylindrical, spherical, and planar patches, respectively:

$$
I=C \cup S \cup P
$$

where $C$ is the set of cylindrical patches, $S$ is the set of spherical patches and $P$ is the set of planar patches. Note that composite patches (such as a patch with both cylindrical and planar surfaces) must be broken up into primitive patches (one of the above three types).

At this stage, when each element of $I$ is a primitive patch, the elements are examined in turn. For a particular element, all boundary segments which are not "lines" in their particular manifolds are discretized into primitive segments (lines are straight lines are on planes, circular arcs and straight lines on a cylinder for constant $z$ and constant $\theta$ respectively, and great arcs are on a sphere). The intersection set $I$ now consists of a finite number of primitive patches, each of which is composed of a finite number of primitive boundary segments.

The non-penetration condition for each end-point or vertex of each primitive boundary segment is written as a constraint in the linear program. The linear program can now be solved using standard LP techniques.

Finding one solution to the simplex is easy; finding all solutions is a more difficult proposition. However, useful information can still be obtained by projecting the simplex on either the $\vec{v}$ or the $\vec{\omega}$ space. Such linear programming methods have previously been used by Mattikalli et al. (1994) to obtain solutions to the stability problem for assemblies.

Solutions are returned in the form of allowable translational and angular velocities. Translational velocities of zero indicate that translation is constrained for that bodybody pair. Angular velocities of zero indicate that rotation is constrained for that body-body pair.

Some post-processing may need to be done, such as realizing that two revolute joints with a common rotation axis actually imply only a single degree of freedom. Once this is done, the low-level representation of the assembly (i.e. the point and line feature representation) can be easily generated. Such a representation specifies the rotation axis and positions of the joints.

\section{Performance of the Linear Program}

When contact patches do not have boundaries for which non-penetration can be expressed by non-penetration at the end-points, it is necessary to discretise such boundaries into segments. Non-penetration at the end-points of such a segment implies non-penetration at each pointon the segment.

However, the number and choice of such segments influence the size and conditioning of the linear program. The end-points must be chosen such that:

1. There are sufficient number of points between them; i.e. the length of the segment must be sufficiently above the numerical accuracy threshold of the solid modeling system.

2. The chosen points are well spaced along the boundary; i.e. segment lengths must be comparable.

\section{Giving Feedback to the Designer}

Useful feedback can be provided to the designer in the form of questions such as: "Which parts can rotate when all translations are constrained?" This question can be answered by adding $\vec{v}+\vec{\omega} \times \vec{p}=\overrightarrow{0}$ with $\vec{p}$ as a point on the body in question, to the set of constraints and evaluating the linear program. This effectively obtains the projection of the linear program on $\vec{v}$. Other possible "what-if" analyses include grounding a part (i.e. setting $\vec{v}=\overrightarrow{0}$ and $\vec{\omega}=\overrightarrow{0}$ for that part) and obtaining the instantaneous DOFs (Degrees Of Freedom) for all the other parts.

Such "what-if" questions are answered by solving the projected simplex. The vertices of this polyhedron can be enumerated to obtain the space of possible solutions.

Alternatively, the 6-dimensional simplex can be solved to obtain the translational and angular velocities for each body-body pair. All those body-body pairs which have zero translational velocity are restricted in translation. Bodybody pairs with zero angular velocity are restricted in rotation. When both are zero, then the body-body pair form a fixed joint.

\section{EXAMPLE}

To demonstrate the progression of our algorithm, we use as an example the assembly shown in Figure 5. This assembly has four parts, which form two revolute joints, one between the inner lever and support 1 and support 2, and 
another between the outer ring and support 1 and support 2 .

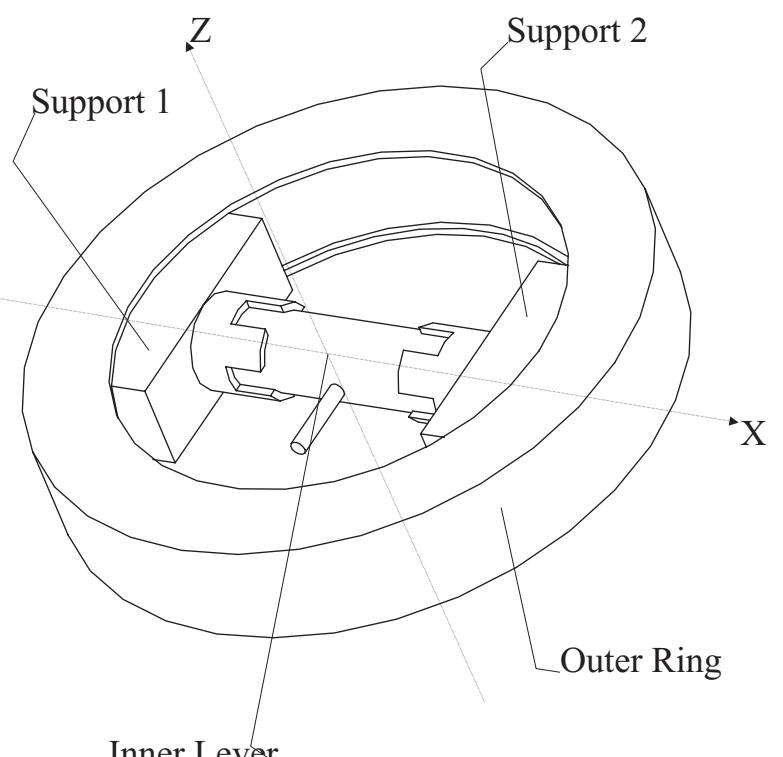

Figure 5. Example Assembly with Four Parts and Two Revolute Joints.

The parts are intersected after scaling them up by a small factor, to obtain manifold bodies after intersection. The contact graph for the assembly is shown in Figure 6 . There are 4 body-body pairs in the assembly, indicated by edges in the graph.

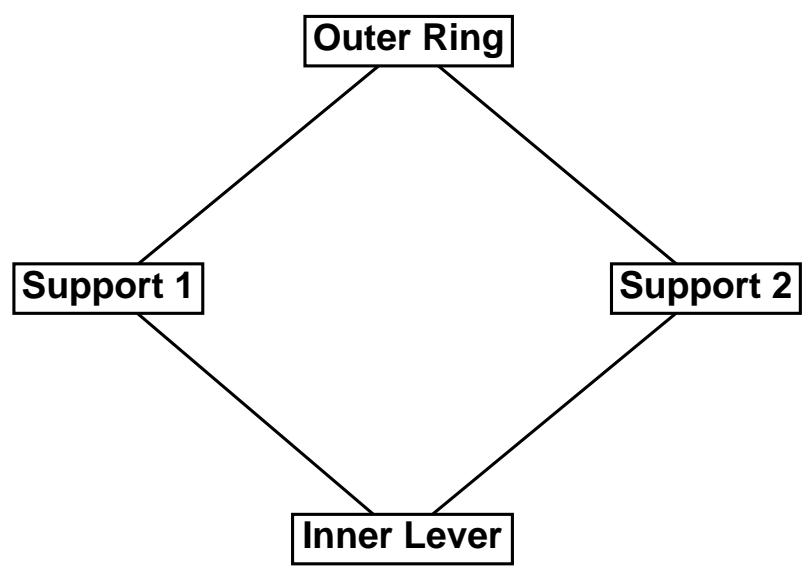

Figure 6. Contact Graph for the Example Assembly.
Upon examining the nature of the contacts between parts (Figure 7), we find that there are 4 compound patches or contacts. These patches are rationalized to 2 planar patches and 14 cylindrical patches.

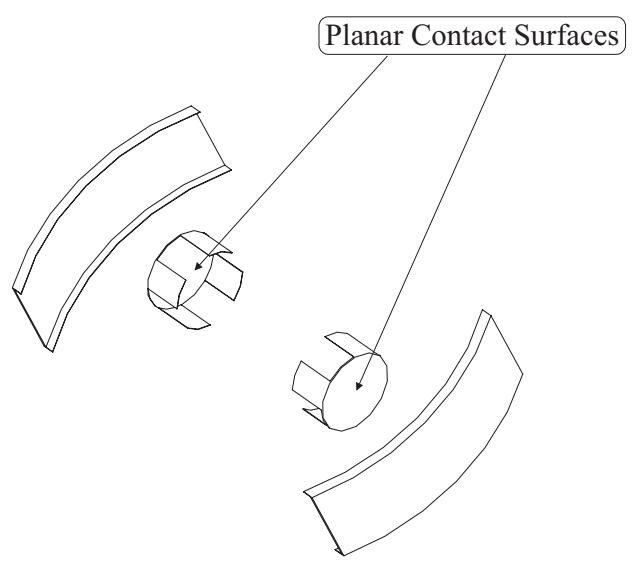

All other surfaces are
Cylindrical Contact Surfaces

Figure 7. Surface Contacts Between Parts.

The boundary of each planar patch is approximated with line segments (say, with $l_{1}$ and $l_{2}$ segments, respectively) to obtain the non-penetration conditions at each vertex of the line segments. This adds a finite set of nonpenetration conditions to the linear program. This is set $P$. The set $P$ has $l_{1}+l_{2}-2$ members.

The cylindrical patches are all bounded by constant $z$ or constant $\theta$ segments. Therefore, an exact set of nonpenetration conditions can be written for each cylindrical patch. One non-penetration condition is contributed by each end point of each constant $z$ and constant $\theta$ segment. This is set $C$. The set $C$ has 56 members (see Figure 7 ).

As a result, the linear program for the entire assembly has $l_{1}+l_{2}+54$ constraints. The solution of this linear program yields values for the translational and angular velocities for each body-body pair. All the body-body pairs are thus constrained in translation. However, all the bodybody pairs are not constrained in rotation. The returned result indicates 4 revolute joints.

Post-processing indicates that that of the 4 revolute joints, there are two pairs, each with a common rotation axis. Therefore the 4 revolute joints actually articulate 2 DOFs.

The rotation axis and the position completely specify 
Table 1. Representation of Revolute Joints in the Example Assembly.

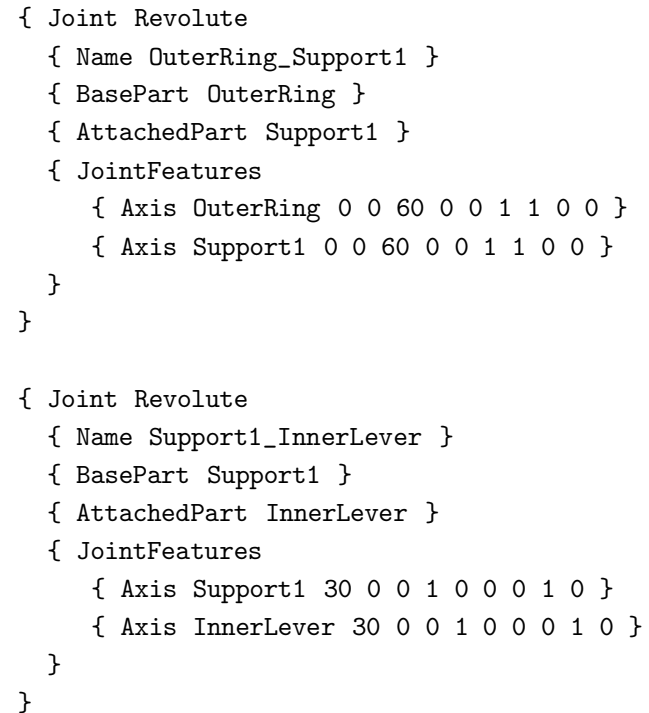

each revolute joint (see Table 1). Other information needed is the pair of parts which form the joint, and a way of knowing which of the parts in the pair is the grounded or base part.

\section{CONCLUSION}

In this paper, we have presented a new methodology for determining the instantaneous degrees of freedom of an assembly. In addition, we have described an algorithm to automatically compute this representation, given an assembly with the geometry and positions of its components. This information is useful feedback for the designer so that he or she is able to identify and resolve any potential problems with under-constrained or over-constrained parts.

Beginning with body to body contact information, we reason about the nature of these contacts to obtain surface mating conditions. Linear non-penetration constraints are written for each of the mating surfaces and solved using linear programming. Knowing the instantaneous degrees of freedom, we obtain a point and line articulation representation. Once this representation is obtained, our implementation can automatically simulate the articulation, and will maintain internal consistency in the assembly model.

This paper concerns itself with planar, cylindrical and spherical contacts only. Our work extends previous results for planar contacts to spherical and cylindrical contacts. Such contacts form the lower pairs of kinematic joints.

\section{ACKNOWLEDGMENT}

Ongoing research in this area is funded in part by DARPA under contract ONR \#N00014-96-1-0854, by the Raytheon Company, the Robotics Institute, and the Institute for Complex Engineered Systems at Carnegie Mellon University. We would like to thank other people in our group who have contributed to the implementation of our system: Antonio Díaz-Calderón and Sripal Mehta.

\section{REFERENCES}

Ambler, A.P.; and Popplestone, R.J., "Inferring the Positions of Bodies from Specified Spatial Relationships", Artificial Intelligence, Vol. 6, 1975, pp. 157-174.

Anantha, R.; Kramer, G.A.; and Crawford, R.H., "Assembly Modelling by Geometric Constraint Satisfaction", Computer-Aided Design, Vol. 28, No. 9, 1996, pp. 707-722.

Baraff, David; and Mattikalli, Raju, "Impending Motion Direction of Contacting Rigid Bodies", Technical Report CMU-RI-TR-93-15, The Robotics Institute, Carnegie Mellon University, Pittsburgh, PA 15213.

Ge, Q.J.; and McCarthy, J.M., "Functional Constraints as Algebraic Manifolds in a Clifford Algebra", IEEE Transactions on Robotics and Automation, Vol. 7, No. 5, October 1991, pp. 670-677.

Kim, S.H.; and Lee, K., "An Assembly Modeling System for Dynamic and Kinematic Analysis", ComputerAided Design, Vol. 21, No. 1, January/February 1989, pp. $2-12$.

Ko, H.; and Lee, K., "Automatic Assembling Procedure Generation from Mating Conditions", Computer-Aided Design, Vol. 19, No. 1, January/February 1987, pp. 3-10.

Liu, Yanxi; and Popplestone, Robin, "A Group Theoretic Formalization of Surface Contact", The International Journal of Robotics Research, Vol. 13, No. 2, April 1994, pp. 148-161.

Mattikalli, Raju; Baraff, David; and Khosla, Pradeep, "Finding All Gravitationally Stable Orientations of Assemblies", Proceedings of the IEEE International Conference of Robotics and Automation, San Diego, May 1994, pp. 251257.

Mattikalli, R.; and Khosla, P.K., "Analysis of Restraints to Translational and Rotational Motion from the Geometry of Contact, DE-Vol. 39, Issues in Design Manufacture/Integration, Proceedings of the ASME Winter Annual Meeting, Atlanta, GA, December 1-6, 1991, pp. 65-71.

Rajan, V.N.; and Nof, S.Y., "Minimal Precedence Constraints for Integrated Assembly and Execution Planning", IEEE Transactions on Robotics and Automation, Vol. 12, No. 2, April 1996, pp. 175-186.

Rajan, Venkat N.; Lyons, Kevin W.; and Sreerangam, Raj, "Generation of Component Degrees of Freedom from 
Assembly Surface Mating Constraints", Proceedings of the 1997 ASME Design Engineering Technical Conference, September 14-17, 1997, Sacramento, California.

Rocheleau, D.N., "An Assembly Representation for Articulated Mechanisms", PD-Vol. 80, Proceedings of the 1996 ASME Engineering Systems Design and Analysis Conference, Vol. 8, 1996.

Sodhi, R.; and Turner, J.U., "Towards Modelling of Assemblies for Product Design", Computer-Aided Design, Vol. 26, No. 2, February 1994, pp. 85-97.

Thomas, F.; and Torras, C., "Inferring Feasible Assemblies from Spatial Constraints", IEEE Transactions on Robotics and Automation, Vol. 8, No. 2, April 1992, pp. 228-239.

Tilove, R.B., "Extending Solid Modeling Systems for Mechanism Design and Kinematic Simulation", IEEE Computer Graphics and Applications, May/June 1983, pp. 9-19.

Turner, J.U.; Subramaniam, S.; and Gupta, S., "Constraint Representation and Reduction in Assembly Modeling and Analysis", IEEE Transactions on Robotics and Automation, Vol. 8, No. 6, December 1992, pp. 741-750.

Wilson, Randall H.; and Latombe, Jean-Claude, "Geometric Reasoning about Mechanical Assembly", Artificial Intelligence, Vol. 71, No. 2, December 1994, pp. 371-396. 